\title{
Comparison Performance of PI and FPI Controllers for Model Reduction of Binary Distillation Column Plant
}

\author{
Nasir Ahmed Alawad, Afaf Jebar \\ Computer Engineering Department, College of Engineering, Mustansiriyah University, Baghdad, Iraq
}

\section{Keywords}

Distillation column,

Optimization methods,

$P I$,

FPI controllers,

MATLAB toolbox.

\begin{abstract}
Distillation is the separation strategy in the oil and compound businesses for cleansing of conclusive items. This paper deals with the reduced dynamic model and control of the distillation tower by applying a multi-loop control framework in Matlab/Simulink for a double blend. The structure objective considered is to guarantee that the top item, the base item, the reflux rate and the reboiler rate stray inside their recommended limits forever and for all the aggravations. This paper proposes an optimal tuning method for fractional Proportional-Integral controller (FPI). The method consists of minimizing Integral Absolute Error (IAE) performance index criterion. Acceptable controller (FPI) is acquired via looking in the space of plan boundaries (Kp,Ki). An example of application (distillation column) plant is presented to evaluate the proposed method. A comparison with classical PI controller and optimal PI shows that the system under fractional state is robust in terms of transient specifications, maximum overshot, settling and rise times. The simulation results shows that more than (21\%) improvement for reducing the max-overshot and(65\%)improvement for increasing response speed for fractional PI compared with classical PI.MATLAB simulation toolbox is used to show the effectives of the proposed method.
\end{abstract}

\section{1-Introduction}

As of late the idea of fractional calculus are generally presented in numerous territories in science and engineering. In control frameworks, this idea are effectively used to develop fractional order controllers. Therefore, the closed-loop control framework exhibitions are improved in examination with old style controllers (PID). Execution of ordinary PI control techniques are suspicious in high immaculateness distillation section. The fundamental downside is the late reaction of the restorative activity particularly that controlled factors (compositions) modifies enthusiastically with the principle unsettling influences (feed stream and piece) as mentioned by (Sigurd Skogestad, 1997[1]). As a result, quality of product is affected and consequently, economical loss to plant is likely. It was also reported by (Shinskey, 1984; S. Skogestad, 2007)[2,3] that the top and base item arrangements tight collaboration which make the procedure touchy to little changes are a major worry in the industry. It ought to be noticed that another gathering of PID controllers with fractional orders have been created lately for viable procedure control frameworks (Tepljakov, et al., 2018) [4]. These fractional order controllers are demonstrated with fractional-order differential conditions. Fractional Proportional-Integral (FOPI) controller is picked for item's structure control of Wood and Berry (WB) segment because of its intrinsic time space adaptability. FOPID controllers can be planned and tuned for both SISO frameworks and MIMO frameworks including time delays (Baruah, et al., 2016; Vale'rio and Costa, 2009; Monje, et al., 2010)[5-7]. The MIMO control framework applications, some notable strategies for tuning this class of controllers exist. These structure strategies are either founded on time area boundary improvement or frequency space. FOPID controller boundaries can be processed legitimately in recurrence area to meet some ideal details, for example, phase margin, consistent stage at gain cross over frequency and other affectability limitations as appeared by a few researchers (Luo, et al., 2010; Luo \&Chen, 2009; Vale'rio \&Costa,2009) [8-9,6]. Different strategies for structuring FOPID controllers incorporate optimal based techniques, for example, Linear Matrix Inequality (LMI), probabilistic streamlining file, and developmental calculations (Lee and Chang, 2010; Song, et al., 2011; Wu, et al., 2018)[10,11,12]. FOPID controller is adaptable as far as time domain trademark. When appropriately tuned, this time area adaptability empowers FOPID controller to yield unrivaled control execution over conventional PID controllers for a class of systems. A few researchers exhibited this similar advantage by reenactment investigations of both FOPID controller and classical PID controllers (Monje, et al., 2010; Lee and Chang, 2010; Padula, et al., 2014)[7,10,13].fractional order control frameworks have been accounted for getting better execution when contrasted with traditional (integer order) controllers under reasonable correlation however to the detriment of additionally tuning boundaries (Li and Chen, 2014)[14].In terms of power to pick up varieties and vulnerabilities, FOPID controller is known to give better execution contrasted with ordinary PID controller when appropriately structured. A significant commitment of this paper is in this way the improvement of structure techniques for multivariable FOPI controllers reasonable for piece control of refining section. Usage of these fractional order controllers is shown by recreation on both twoby-two distillation systems. This paper gives an improvement in transient response, less overshot and quickly response to reach the desired composition of distillation column when compared with my previous work (Jaber and Alawad).

2-Mathematical Model of WB Distillation Plant 
Refining is one of the most significant unit activities in substance engineering. The point of a refining section is to isolate a blend of parts into at least two results of various organizations. The separation happens in a vertical segment where warmth is added to a reboiler at the base and expelled from condenser at the top. The first $2 \times 2$ MIMO process is presented by (Wood and Berry, 1973)[15]. The process transfer function matrix of the distillation process is given by:-

$\mathrm{G}(\mathrm{s})=\left[\begin{array}{cc}\frac{12.8 e^{-s}}{16.7 s+1} & \frac{-18.9 e^{-3 s}}{21 s+1} \\ \frac{6.6 e^{-7 s}}{10.9 s+1} & \frac{-19.4 e^{-3 s}}{14.4 s+1}\end{array}\right]$

In this system, it is required to control the top composition (Xd) and bottom composition $(\mathrm{Xb})$, by manipulating reflux flow rate(R) and vapor flow rate(V)respectively. There are large interaction between the two loops, so that needs decoupler. In this work, the simplified decoupler is used, because it is simple and widely used in the literature (Luyben, 1970)[16].The decoupler is:-

$D(s)=\left[\begin{array}{cc}1 & \frac{-g_{12}}{g_{11}} \\ \frac{-g_{21}}{g_{22}} & 1\end{array}\right]$

So that, equ(2),can be written as:-

$\mathrm{D}(\mathrm{s})=\left[\begin{array}{cc}1 & \frac{1.476(16.7 s+1) e^{-2 s}}{16.7 s+1} \\ \frac{0.33(14.2 s+1) e^{-4 s}}{10.89 s+1} & 1\end{array}\right]$

The resulting transfer matrix $T(s)$ is then:

$T(s)=\left[\begin{array}{cc}g_{11}(s)-\frac{g_{12}(s) g_{21}(s)}{g_{22}(s)} & 0 \\ 0 & g_{22}(s)-\frac{g_{12}(s) g_{21}(s)}{g_{11}(s)}\end{array}\right]$

Then, according to equation (4), the diagonal matrix of WB column is given by:-

$\overline{g_{11}}(s)=\frac{12.8 e^{-s}}{16.7 s+1}-\frac{6.237(14.36 s+1) e^{-7 s}}{228.69 s^{2}+31.89 s+1}$

$\overline{g_{22}}(s)=\frac{-19.4 e^{-3 s}}{14.4 s+1}+\frac{9.745(16.7 s+1) e^{-9 s}}{228.69 s^{2}+31.89 s+1}$

This choice makes the realization of the decoupler easy, but the diagonal transfer matrix $T(s)$ obtained is complex since its elements are the sum of transfer functions. Controller tuning can therefore be difficult. It is then often suggested to approximate each sum by a simpler transfer function to facilitate controller tuning.

\section{3-Model Reduction of WB Distillation Plant}

Model reduction is a technique widely used in part of dynamic analysis and design of structures. (Ramakrishnan et al., 1989) [17] proposed Routh expansion method for reducing the order of a flexible system, the procedure does not require the knowledge of the system eigenvalues/eigenvectors and guarantees a stable reduced-order model if the original system is stable. (Sidi-Ali-Cherif et al. 1999) [18] Examined the use of Krylov-subspace iterative methods to obtain low-rank approximate solutions of Lyapunov equations for use in model reduction of large space structures. Generally for MIMO systems (distillation column), there are Various methods were proposed to approximate the diagonal elements of the decoupled matrix (g11, g22) to some standard forms (Arkun, et al., 1984; Wood and Berry,1973; Shinskey.et al,1988)[19,15,20]. However, all of them only deal with integer order transfer functions.
The Pade approximation is often used to approximate a pure time delay by a rational transfer function, so that equations (5) and (6) can be approximated and remove the nonlinear term in these equations by using (Kalpana,et al.,2017) [21]:-

$\mathrm{e}^{-\theta} \underset{\mathrm{s}}{ } \frac{1-\frac{\theta}{2} s}{1+\frac{\theta}{2} s}$

Where $(\theta)$ is the delay term

$\mathrm{G}_{11 \mathrm{~W}}=\frac{-1432 s^{4}+7368 s^{3}+1843 s^{2}+155.2 s+3.75}{3819 s^{5}+9491 s^{4}+3971 s^{3}+547.1 s^{2}+30.05 s+0.5714}$

$\mathrm{G}_{22 \mathrm{~W}}=\frac{2093 s^{4}-2698 s^{3}-710.1 s^{2}-59.71 s-1.43}{3293 s^{5}+3615 s^{4}+1146 s^{3}+144.1 s^{2}+7.747 s+0.1481}$

It is seen that, they obtained equations (8) and (9) are of high order and these made complex and difficult to use so model reduction techniques is used to reduce the order of these equations.

In this work, an optimization method can be used for more accuracy to get reduced transfer function of the two loops $\left(\mathrm{G}_{11 \mathrm{w}}\right)$ and $\left(\mathrm{G}_{22 \mathrm{w}}\right)$. Particle Swarm Optimization (PSO) method has proved to be excellent optimization tool in the past few years. The result of applying PSO algorithm to equation (8) and (9), for more details see (Afaf.et al, 2020) [22] are :-

$\mathrm{G}_{11 \mathrm{rWB}}=\frac{0.8498 s+0.5051}{s^{2}+0.9989 s+0.077}$

$\mathrm{G}_{22 \mathrm{rWB}}=\frac{0.7324 s-1}{0.7811 s^{2}+1.047 s+0.1049}$

\section{4- FPI controller design}

In this paper, Decentralized Decoupling Method control strategy is used, where the proposed algorithm involves combination of simplified decoupler, and decentralized controller (FPI) design with model reduction of the effective transfer function, for the models under test.Fig.1. Shows decentralized decoupling control system.

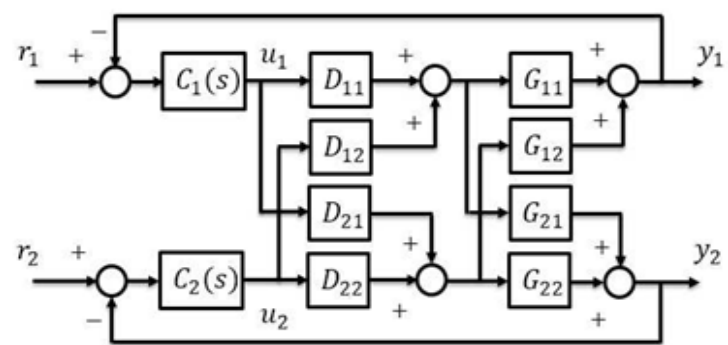

Figure 1. Decentralized decoupling control system

\subsection{Classical PI controller}

Design of PI controller by using PIDtune MATLAB statement for Wood and Berry (G11rWB and G22rWB) is tabulated in Table 1. In this state to evaluate the PID controllers, simulations experiments were conducted by setting the desired product compositions to ( $\mathrm{Xd}=0.98)$ and $(\mathrm{Xb}=0.02)$ (mole fraction) for the distillate and bottoms product, respectively. 
Table 1. PID controller gains for G11rW and G22rW

\begin{tabular}{lccc}
\hline To control & $\mathrm{Kp}$ & $\mathrm{Ki}$ & $\mathrm{Kd}$ \\
\hline $\begin{array}{l}\text { Distillate Composition (Xd) for } \\
\text { G11rw }\end{array}$ & 0.0764 & 0.0248 & - \\
Bottoms Composition(Xb) for & -0.163 & 0.0286 & - \\
G22rW & & & \\
\hline
\end{tabular}

\subsection{PI control design by optimization algorithms}

The optimal parameters of the PID $\left(k_{p}, k_{i}, k_{d}\right)$ can be obtained by applying the Differential Evolution (DE) algorithm and Invasive Weed Optimization (IWO) algorithms, which are depended on the fitness function (performance index). Generally, there are many types of performance index like:

$I S E_{r}=\int_{0}^{\infty} \mathrm{e}^{2}(t) d t$

$I A E=\int_{0}^{\infty}|\mathrm{e}| d t$

$I T A E=\int_{0}^{\infty} t|\mathrm{e}| d t$

$\operatorname{ITSE}=\int_{0}^{\infty} t \mathrm{e}^{2}(t) d t$

Where ISE $_{\mathrm{r}}$ is integral square error, IAE is integral absolute error, ITAE is integral time absolute error, and ITSE is integral time square error. In this work, the performance index in Eq. (13) is adopted to find the fitness of each solution in population size for both DE and IWO algorithms.

Our objectives are to adjust the three fractional PI parameters (Kp, Ti , and $\lambda$ ) that minimizing IAE defined by the objective function .Eq(13).

In (Storn, et.al,1997) [23] proposed a new floating point encoded evolutionary algorithm for global optimization and named it DE owing to a special kind of differential operator, which they invoked to create new offspring from parent chromosomes instead of classical crossover or mutation. Steps of DE are given as (Trais, et.al,2013[24]. In this work, the parameters of the $\mathrm{DE}$ is determined by trial and error with its specific ranges are listed in Table 2.

Table 2. The DE algorithm parameters.

\begin{tabular}{lc}
\hline Parameters & Value \\
\hline Problem dimension (D) & 5 \\
Population size (NP) & 25 \\
Crossover constant (CR) & 0.6 \\
Scaling constant (F) & 0.95 \\
Number of generation (GEN) & 20 \\
\hline
\end{tabular}

The values of DE-PI controllers are $\mathrm{Kp}=24.501$ and $\mathrm{Ki}=24.601$ for $\mathrm{G} 11 \mathrm{rw}, \mathrm{Kp}=-0.2717$ and $\mathrm{Ki}=-0.028$ for $\mathrm{G} 22 \mathrm{rW}$.

IWO algorithm was first introduced in (Mehrabian, et.al,2006) [25]. It was inspired from colonization of weeds. The behavior of invasive weeds in a cropping system is as follows: (Thotakura,2015) [26]

Weeds invade the fields by dispersing their seeds with the help of air These seeds occupy the available spaces and grow into flowering weeds by utilizing the available resources. Those weeds that adap well to the environment produce more seeds than others. The IWO is explained with the help of a flowchart in Fig.2.

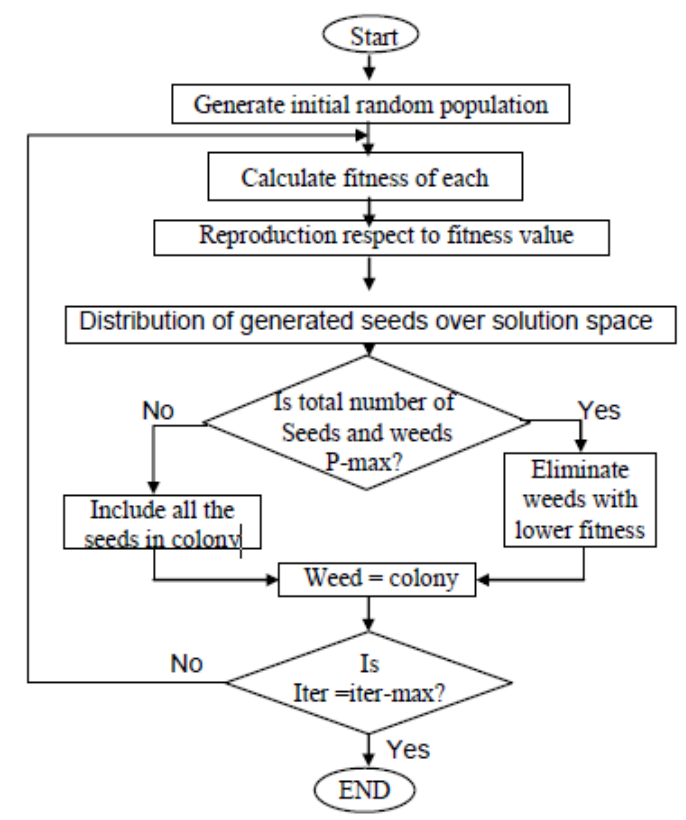

Figure 2. Flowchart of IWO algorithm

In this work, the parameters of the IWO is determined by trial and error with its specific ranges are listed in Table 3.

Table. 3 The IWO algorithm parameters.

\begin{tabular}{ll}
\hline Parameters & Value \\
\hline Number of Generation & 20 \\
Initial Population Size & 2 \\
Maximum Population Size & 10 \\
Minimum Number of Seeds & 0 \\
Maximum Number of Seeds & 5 \\
Initial Value of Standard Deviation & 0.5 \\
Final Value of Standard Deviation & 0.001 \\
\hline
\end{tabular}

The values of IWO-PI controllers are $\mathrm{Kp}=5.1002$ and $\mathrm{Ki}=5.0121$ for G11rw, Kp=-0.5101 and $\mathrm{Ki}=-0.046$ for $\mathrm{G} 22 \mathrm{rW}$.

\subsection{FPI controller}

One of the possibilities to improve PID controllers is to use fractionalorder controllers with non-integer derivation and integration parts.FOPID controller may be defined in continuous form as given below:

$C(s)=K_{p}+\frac{K_{i}}{s^{\lambda}}$

Where $\mathrm{Kp}$ is the proportional gain, $\mathrm{Ki}$ is integral gain and $(\lambda)$ is the fractional integration action order. It is required to find the optimal values of the FOPI controller $\left(K_{p}, K_{i}, \lambda\right)$ to minimize the IAE performance index using IWO and DE. Table 4. Shows the values for G11rw.

Table .4 The optimal parameters of FOPI controller for G11rw and performance index IAE

\begin{tabular}{lcccc}
\hline $\begin{array}{l}\text { Controller } \\
\text { type }\end{array}$ & $K_{p}$ & $K_{i}$ & $\wedge$ & $\begin{array}{c}\text { Average No. } \\
\text { of Iteration }\end{array}$ \\
\hline DE-FOPI & 25.9508 & 24.9972 & 1.1 & 15 \\
IWO-FOPI & 7.5578 & 2.0412 & 0.8579 & 15 \\
\hline
\end{tabular}


And Table 5. The optimal parameters of FOPI controller for G22rW by using performance index IAE

Table .5 The optimal parameters of FOPI controller for G22rW and performance index IAE

\begin{tabular}{lcccc}
\hline $\begin{array}{l}\text { Controller } \\
\text { type }\end{array}$ & $K_{p}$ & $K_{i}$ & $\wedge$ & $\begin{array}{c}\text { Average No. } \\
\text { of Iteration }\end{array}$ \\
\hline DE-FOPI & -0.2799 & -0.029 & 0.9861 & 15 \\
IWO-FOPI & -0.4 & -0.04 & 1.021 & 15 \\
\hline
\end{tabular}

\section{Simulation Results and Comparison}

To appear the effectiveness of FPI controller, when compared with classical and optimized PI, the MATLAB simulation can be used. Tables 6. And 7. shows the transient response analysis results of G11rw and G22rW systems for IAE performance index.

Table 6. Transient response analysis results of G11rw system for IAE

\begin{tabular}{cccccc}
\hline $\begin{array}{c}\text { controller } \\
\text { Type }\end{array}$ & $M p$ & $T_{s}$ & $T_{r}$ & $e_{S S}$ & Under \\
shoot \\
\hline $\begin{array}{c}\text { MTLAB Tuner- } \\
\text { PID } \\
\text { DE-PI }\end{array}$ & 12.38 & 46.69 & 13.86 & 0 & 0 \\
IWO-PI & 2.372 & 0.629 & 0.097 & 0 & 0 \\
DE-FOPI & 2.965 & 3.180 & 0.388 & 0 & 0 \\
IWO-FOPI & 0.577 & 0.688 & 0.350 & 0 & 0 \\
\hline
\end{tabular}

Table 7. Transient response analysis results of G22rW system for IAE

\begin{tabular}{lccccc}
\hline $\begin{array}{l}\text { Controller } \\
\text { Type }\end{array}$ & $M_{p}$ & $T_{s}$ & $T_{r}$ & $e_{s s}$ & $\begin{array}{c}\text { Under } \\
\text { shoot }\end{array}$ \\
\hline $\begin{array}{l}\text { MTLAB Tuner- } \\
\text { PID }\end{array}$ & 9.4941 & 26.3414 & 6.0747 & 0 & 3.7694 \\
DE-PI & 0 & 7.7918 & 4.3258 & 0 & 6.2333 \\
IWO-PI & 17.926 & 11.3792 & 1.7423 & 0 & 12.1463 \\
DE-FOPI & 0 & 7.496 & 4.1382 & 0 & 6.4527 \\
IWO-FOPI & 9.0924 & 8.638 & 2.4027 & 0 & 9.4128 \\
\hline
\end{tabular}

Fig. 3 and fig. 4 shows the comparison between all transient response of PI controllers for G11rw and G22rW systems.

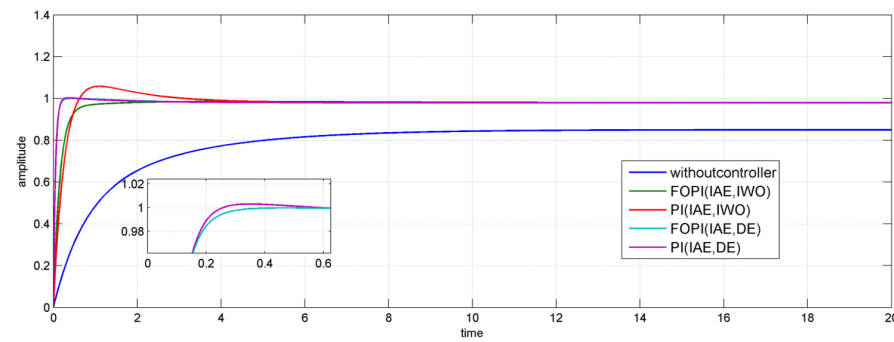

Figure 3. Step Response comparison of all Controllers using IAE for G11rw

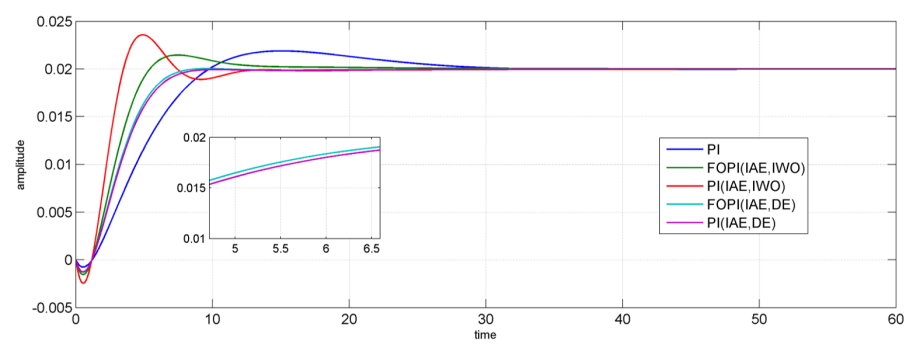

Figure 4. Step Response comparison of all Controllers using IAE for G22rW

It shows that PI controller's performance suffers, in terms of reaching the set-point for both product compositions with some oscillations and large settling time. However, after 46.7 minutes the PID controller for Xd managed to reach the set-points, and the steady state errors reached zero or satisfied the desired composition (0.98 mole fraction), while after 26.3 minutes the PI controller for $\mathrm{Xb}$ managed to reach the set-points, and the steady state errors were reduced until reached zero or satisfied the desired composition (0.02 mole fraction). It is clear that, the response is sluggish and some overshot.

Fig.3 and Fig4. illustrates the responses of the DE-PI and IWO-PI controllers when the performance index is IAE. As shown in these figures, the system with results in less overshoot, less rise time and settling time for both $\mathrm{DE}$ and IWO, it gives also best result, when compared with the MATLAB-PI. The DE-PI shows best result, when compared with PID-IWO as shown in Table.6 and Table.7.

Fig.4 shows for all controllers, there are undershoots due to the G22rw system is non-minimum phase (there is a zero on the right-hand side of S-plane), but generally is small in DE-PI when compared with IWOPI controller. The classical MATLAB PI controller, gives the smallest undershot, but high settling time (very sluggish response).

\section{Conclusion}

This paper compared the performance of classical PI, fractional PI, and optimal PI controllers within the context of a typical process control environment. An optimal design method for fractional PI controller has been presented. The method is based on using optimal representation of fractional operator. The optimal settings have been obtained by minimizing IAE using Matlab optimization toolbox. The simulation results have shown the effectiveness of the proposed method in comparison with classical PI controller. In addition, the obtain controlled system is robust, when compared with optimal PI controller. A single objective optimization algorithm (IWO, DE) has been employed to study the performance for different of controller structures. This also provides more flexibility and opportunity to better adjust the dynamical properties of the control system. The fractional order controller revels good robustness. Model reduction procedures in process control activity is one of the significant issues, likewise the linearization of distillation plant is valuable, when the computational power utilized in controlling of such activity is big. Fractional order PI controller for integer order system offer better flexibility in adjusting gain and phase characteristics than the PID controllers, owing to the extra tuning parameter i.e. order of 
integration in addition to proportional gain, integral time. FOPID controller gives a small instant negative undershoot, which is undesirable state, due to the non- minimal phase system G22rw (there is a zero in the right-hand side of s-plane). Generally, there are more than (21.455\%) improvement in (MP) for IWO-FPI, when compared with classical PI controller and about speed response (Ts), there is more than (67.86\%) improvement in IWO-FPI, when compared. In the future work using intelligent controllers (fuzzy and neural) and compared with this fractional PI controller.

\section{References}

[1.] Skogestad, S., Dynamics and control of distillation columns-a critical survey. Modeling. Identification and Control

\section{8(1997) 177-217.}

[2.] Shinskey, F. G., Distillation control: for productivity and energy conservation: McGraw-Hill (1984).

[3.] Skogestad, S., The Dos and Don'ts of Distillation Column Control Chemical Engineering Research and Design 85(2007)13-23.

[4.] Tepljakov, A. et al., FOPID Controllers and Their Industrial Applications: A Survey of Recent Results. IFAC-PapersOnLine 51(2018)25-30

[5.] Baruah, G., Majhi, S. \& Mahanta, C., Fractional order PID controller design for an SOPDT Model by online tuning method. Hyderabad,India . IEEE Xplore/Indian Control Conference 2016 (2016) 450-455.

[6.] Vale'rio, D. \& Costa, J. S. d., Tuning of Fractional PID Controllers with Ziegler Nichols Type Rules. Signal Processing 86(2009)2771-2784.

[7.] Monje, C. et al., Fractional-order Systems and Controls: Fundamentals and Applications. New York: Springer-Verlag (2010).

[8.] Luo, Y. \& Chen, Y., Fractional order [proportional derivative] controller for a class of fractional order systems. automatica 45 (2009) 2446-2450.

[9.] Luo, Y., Chen, Y., Wang, C. Y. \& Pi, Y. G., Tuning fractional order proportional integral controllers for fractional order system. Journal of Process Control 20(2010)823-831.

[10.] Lee, C.H. \& Chang, F.K., Fractional-order PID controller optimization via improved electromagnetism-like algorithm. Expert Systems with Applications 37(2010) 8871-8878.

[11.] Song, X., Chen, Y., Tejado, I. \& Vinagre, B. M., Multivariable Fractional PID Controller Design via LMI approach. Milan. IFAC (2011) 13960-13965.

[12.] Wu, Z. et al., Tuning for Fractional Order PID Controller based on Probabilistic Robustness. IFAC-PapersOnLine 51(2018) 675-680.

[13.] Padula, F., Vilanova, R. \& Visioli, A., H-infinity optimization based fractional order PID controllers design. International Journal of Robust and Nonlinear Control 24(2014) 3009-3026.

[14.] Li, Z. \& Chen, Y., Ideal, Simplified and Inverted Decoupling of Fractional Order TITO Processes. Cape-Town, IFAC (2014) 2897-2902.

[15.] Wood, R. K., Berry M. W, Terminal composition control of a binary distillation column.Chem. Eng. Sci. 28(1973) 1707-1717.

[16.] Luyben, W.L.," Distillation decoupling". AIChE Journal 16(1970) 198-203.

[17.] Ramakrishnan, J.V., Rao, S.V. and Koval, L.R. 'Reduced-order modeling of flexible structures'. Journal of Guidance, 5 (1998) 459464.
[18.] Sidi-Ali-Cherif, S., Grigoriadis, K.M. and Subramaniam, M. 'Model reduction of large-scale systems using approximate component cost analysis'. Proceedings of the American Control Conference 6 (1999) 4471-4475.

[19.] Arkun, Y., Manouslouthakis, B. and Palazoglu, A., Robustness analysis of process control systems. A case study of decoupling control in distillation. I\&EC Process Design and Development 23(1984) 93-101.

[20.] Shinskey, F.G., Process Control Systems: Application, Design and Adjustment. McGraw-Hill, New York, (1988).

[21.] Kalpana R. , Harikumar K. , Senthilkumar J. , Balasubramanian G. , and Abhay S. Gour," Multivariable Static Output Feedback Control of a Binary Distillation Column using Linear Matrix Inequalities and Genetic Algorithm". Control and System Engineering (2017)1-11.

[22.] Jebar, A., Alawad, N.A., Decoupling and Model Reduction for the Binary Distillation Column Linear System. International Journal of Scientific Engineering and Science 4 (2020) 25-31.

[23.] Storn ,R. , Price, K., Differential Evolution - A Simple and Efficient Heuristic for Global Optimization over Continuous Spaces. Journal of Global Optimization 11(1997) 341-359.

[24.] Andromeda, T., Yahya, A.,et.al, Differential Evolution For Optimization Of Pid Gain In Electrical Discharge Machining Control System. Transactions of the Canadian Society for Mechanical Engineering 37(2013)293-301.

[25.] Mehrabian, A. R., \& Lucas, C., A novel numerical optimization algorithm inspired from weed colonization. Ecological Informatics 1(2006) 355-366.

[26.] Thotakura T., Ramakrishna, et al., Invasive Weed Optimization (IWO) Algorithm for Control of Nulls and Side lobes in a Concentric Circular Antenna Array (CCAA). International Journal of Computer Applications 126(2015)44-49.

\section{How to Cite This Article}

Alawad, N., Jebar, A., Comparison Performance of PI and FPI Controllers for Model Reduction of Binary Distillation Column Plant, Brilliant Engineering, 1(2021), 25-29. https://doi.org/10.36937/ben.2021.001.005 